\title{
Wissensmetropole Ruhr: Einführung in das Themenheft
}

\author{
Matthias Kiese ${ }^{1}$ \\ Online publiziert: 25. April 2019 \\ (c) Deutscher Verband für Angewandte Geographie (DVAG) 2019
}

\section{The Ruhr: Knowledge Metropolis - Introduction to the Special Issue}

Bilder für die Ewigkeit: Am 21. Dezember 2018 wurde dem Bundespräsidenten Steinmeier bei der zentralen Abschiedsveranstaltung für den deutschen Steinkohlenbergbau in Bottrop symbolisch das letzte Stück in Deutschland geförderter Steinkohle übergeben. Nach sechs Jahrzehnten des sozial abgefederten Strukturwandels waren ein bedeutendes Kapitel deutscher Industriegeschichte zu Ende gegangen und die Energiebasis dieser vormals stolzen Industrieregion aufgegeben.

\section{Glückauf Zukunft!}

Unter diesem Slogan beschworen der Bundespräsident und NRW-Ministerpräsident Laschet in ihren Reden die Zukunft des Ruhrgebiets mit Hochschulen, Forschungseinrichtungen und Unternehmensgründungen als neuen Motoren für Beschäftigung und Wohlstand. Darin wird eine Zukunft des Ruhrgebiets als Wissensmetropole gezeichnet, die in den Marketingkampagnen der Region und ihrer Städte bereits fest verankert ist. In einer gemeinsamen „Resolution Wissenschaftsregion Ruhr“ verständigten sich alle maßgeblichen regionalen Organisationen auf das Leitbild, Wirtschaft und Städte in der Metropole Ruhr durch eine starke Wissenschaftsregion zu stärken. Dafür gilt es Bildungsgerechtigkeit herzustellen, den Wissenschafts- und Wirtschaftsstandort zu stärken und die Gründungsmentalität zu fördern (IHK Ruhr et al. 2015). Mit diesen Fragen beschäftigt sich das Forschungsfeld Wissensmetropolen im Kompetenzfeld Metropolenforschung der Universitätsallianz Ruhr, aus dem dieses Themenheft hervorgegangen ist (UAR 2019).

Prof. Dr. Matthias Kiese

matthias.kiese@rub.de

1 Geographisches Institut, Ruhr-Universität Bochum, Bochum, Deutschland

\section{Warum Wissensmetropolen?}

Metropolregionen sind aufgrund ihrer Dichte von Wissensträgern und Netzwerkbeziehungen Kristallisationskerne der Wissensökonomie (vgl. Kujath 2005). Spontane Face-toFace-Interaktionen ermöglichen hier den Austausch auch von neuestem, noch nicht kodifizierten Wissen, das in lokalisierten Lernprozessen zu Wertschöpfung und Beschäftigung durch neue Produkte, Herstellungsverfahren und Dienstleistungen führen kann (vgl. Florida et al. 2017). In Erweiterung der Definition der Wissensökonomie der OECD (1996) lässt sich eine Wissensmetropole als urbane Agglomeration verstehen, deren gesellschaftliche und wirtschaftliche Entwicklung von ihrer Fähigkeit zur Erzeugung und Anwendung bzw. Nutzung sowie zur Aufnahme und Verbreitung von Wissen abhängt. Darin sind die grundlegenden Strategien wirtschaftlicher Entwicklung nach Romer (1993) enthalten: ,producing ideas“ durch Erzeugung und Anwendung sowie ,using ideas“ durch Akquisition und Diffusion.

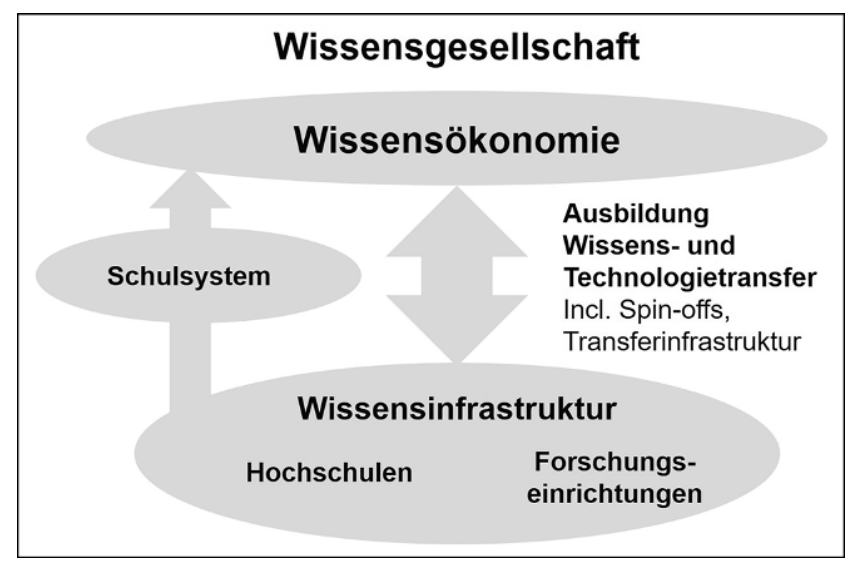

Abb. 1 Wissensmetropole - schematische Darstellung. (Quelle: Eigener Entwurf) 
Als offenes und komplexes System besteht eine Wissensmetropole aus verschiedenen Subsystemen, deren $\mathrm{Zu}$ sammenspiel die Leistungsfähigkeit des großen Ganzen bestimmt (Abb. 1). Der Wissensinfrastruktur aus Hochschulen und Forschungseinrichtungen stehen wissensintensive Industrie- und Dienstleistungsunternehmen gegenüber, die technologisches Wissen und Marktimpulse in Wertschöpfung und Beschäftigung umsetzen. Wissensinfrastruktur und Wissensökonomie sind durch den Bildungssektor mit seinen allgemein- und berufsbildenden Schulen sowie durch verschiedene Kanäle und Infrastrukturen des Wissens- und Technologietransfers miteinander verknüpft. All diese Elemente wiederum sind in den weiteren Kontext der Wissensgesellschaft eingebettet. Mit diesem Begriff umschreibt Stehr (1994) die Durchdringung aller Lebensbereiche mit wissenschaftlichem Wissen, das damit zur zentralen Grundlage von gesellschaftlicher Macht wird (vgl. Stehr/Adolf 2018).

\section{Beiträge im Themenheft}

Der Wandel zu Wissensökonomie und Wissensgesellschaft ist ein Megatrend, der alle urbanen Agglomerationen betrifft. Für das Ruhrgebiet, das den Schatten der montanindustriellen Vergangenheit noch immer nicht überwunden hat, stellt er aber eine spezielle Herausforderung dar. Die frühere Zechenhauptstadt Bochum eignet sich den Wandel als Kernkompetenz an und stellt sich dieser Herausforderung mit besonderer Energie, Strategie und Netzwerken. Lars Tata hat das lokale Netzwerk UniverCity mit Stadt, Hochschulen und IHK gemanagt und koordiniert als Referent für strategische Entwicklung der Stadt die Entwicklung der Bochum-Strategie. Im Interview mit Matthias Kiese spricht er über Ziele und Erfahrungen der Netzwerkarbeit und Strategieentwicklung auf dem Weg zu einer Wissensstadt.

Der Beitrag von Matthias Kiese bewertet die Ausstattung des Ruhrgebiets mit Hochschulen und Forschungseinrichtungen als zentrale Ausgangsbedingung für eine regionale Wissensökonomie sowie deren Entwicklungsstand. Obwohl die Region über eine dichte Hochschullandschaft und eine große Studierendenpopulation verfügt, sind die Hochschulen schlecht ausgestattet und andere Regionen weisen mehr außeruniversitäre Forschungseinrichtungen auf. Gleiches gilt für den Entwicklungsstand der Wissensökonomie, hier zeigen sich anhaltende Defizite in Forschung und Entwicklung, Kreativwirtschaft und wissensbasierten Unternehmensgründungen. Innovative Förderkonzepte brauchen Zeit, um sich in einem messbaren Aufholprozess der Wissensökonomie niederzuschlagen.

Von Hochschulen und außeruniversitären Forschungseinrichtungen in Wissensmetropolen werden wichtige Innova- tionsimpulse erwartet. Am Beispiel der Metropole Ruhr untersuchen Bernd Kriegesmann, Matthias Böttcher und Torben Lippmann anhand von Kooperationsbeziehungen zwischen Wissenschaft und Wirtschaft sowie anhand von Unternehmensgründungen aus der Wissenschaft, welche Effekte in diesem Sinn vor Ort wirksam werden. Im Ergebnis zeigt sich, dass die Kooperationsbeziehungen auf regionaler Ebene nur relativ schwach ausgeprägt sind. Auch Existenzgründungen aus der Wissenschaft finden im Ruhrgebiet im Vergleich zu anderen Metropolräumen in Deutschland seltener statt, womit das vorhandene Innovationspotenzial bislang noch nicht vollständig ausgeschöpft wird.

In Wissensmetropolen fällt Universitätsprofessoren als Generatoren und Multiplikatoren von Wissen als Grundstoff für Innovationen eine wichtige Rolle zu. Basierend auf einer umfangreichen deutschlandweiten Befragung von gut 2900 Hochschullehrern zum Wissenstransfer aus Hochschulen untersuchen Christian Warnecke und Simon Roh$d e$, wie sich die Professoren der drei großen Universitäten des Ruhrgebiets im Vergleich zu ihren Kollegen an anderen deutschen Universitäten als Akteure des Wissenstransfers verstehen und verhalten. Die Ergebnisse zeigen große Übereinstimmungen, aber auch einige signifikante Unterschiede auf. Die Autoren identifizieren noch ungenutzte Potenziale und zu bewältigende Herausforderungen.

Neben dem Silicon Valley gilt Israel und insbesondere Tel Aviv international als besonders erfolgreiches unternehmerisches Ökosystem. Die sogenannte Start-Up-Nation Israel verfügt über die höchste Start-Up-Dichte pro Einwohner. $\mathrm{Zu}$ den Erfolgsfaktoren dieses Booms gehören u.a. das inspirierende Umfeld, die lebensfrohe Atmosphäre, die Rolle des Militärs bei der Fachkräfteausbildung, die Risikobereitschaft und eine hohe Umsetzungsdynamik nach dem Motto „Just Do It“. Simon Rohde, Christian Warnecke und Laura-Fee Wloka sehen die sonnenverwöhnte Stadt am Mittelmeer nicht als Blaupause für das Start-Up-Ökosystem im Ruhrgebiet. Stattdessen untersuchen die Autoren, wo Unterschiede und Gemeinsamkeiten Komplementaritäten erzeugen, von denen beide Ökosysteme gegenseitig durch Vernetzung und Kooperation profitieren können. $\mathrm{Zu}$ diesem Zweck stellen sie in ihrem Beitrag beide Regionen vor und betrachten die Vernetzungsaktivitäten der regionalen Wirtschaftsförderungsgesellschaft Business Metropole Ruhr mit Tel Aviv.

Seit den 1980er-Jahren wurden Technologieparks in vielen Wissensmetropolen als damals neues Infrastrukturinstrument aufgebaut, um Wissens- und Technologietransfer durch die räumliche Nähe von Wissenschaft und Unternehmen zu fördern. Diese Nähe soll wissensintensive Unternehmen bei der Erzeugung, Verbreitung und Anwendung von Wissen in Lern- und Innovationsprozessen unterstützen. In Deutschland stellt der Wissenschafts- und Technologiecampus (WTC) Dortmund eine der ersten Umsetzungen 
dar. Die Erfahrungen zeigen jedoch, dass räumliche Nähe als vernetzungsfördernde Maßnahme allein nicht ausreicht, sondern durch weitere Formen der Nähe und andere Förderansätze ergänzt werden muss. Elena-Franziska Schlich geht in ihrem Beitrag der Frage nach, inwieweit die Integration ergänzender Maßnahmen in traditionellen Technologieparks wie dem des WTC erfolgte und inwieweit sie zu dessen Zukunftsfähigkeit beitragen. Die Autorin stellt fest, dass eine unreflektierte Übernahme von Literaturempfehlungen und daraus abgeleitete implizite Wirkungsannahmen teils zu negativen Auswirkungen auf im WTC ansässige Organisationen führen können.

Der abschließende Beitrag von Sebastian Jeworutzki und Jörg-Peter Schräpler widmet sich den Schulen als Subsystem der Wissensmetropole Ruhr. Ihre kleinräumige Längsschnittanalyse zeigt, dass sich viele ehemalige Arbeiterbezirke im Norden des Ruhrgebiets im Zuge des Strukturwandels mittlerweile zu sozial benachteiligten Bezirken entwickelt haben. Anhand der Analyse von Übergängen von der Grundschule zum Gymnasium und mithilfe von multivariaten statistischen Modellen zeigen die Autoren, dass die sozialen Disparitäten auch zu einer Verringerung der Bildungsbeteiligung der Kinder in diesen Bezirken führen.

Wissensinfrastruktur und Wissensökonomie, Technologieparks, Forschungskooperationen, Start-ups und Entrepreneurship-Ökosysteme, Professoren als Akteure des Wissenstransfers, soziale Mobilität durch Bildung, internationale Kooperationen: Die in diesem Themenheft versammelten Beiträge untersuchen beispielhaft für das Ruhrgebiet ganz unterschiedliche Subsysteme und Facetten von Wissensmetropolen. Beim Übergang zu Wissensökonomie und Wissensgesellschaft stehen alle Stadtregionen vor ähnlichen
Herausforderungen. Im Ruhrgebiet treten sie aufgrund der altindustriellen Vorprägung besonders deutlich hervor und lassen sich gewissermaßen unter einem Brennglas studieren. Die Autoren dieses Themenhefts hoffen, damit allen Lesern wertvolle Einsichten in die wissens- und innovationsbasierte Entwicklung von Städten und Regionen zu bieten. Wir wünschen eine anregende Lektüre!

Interessenkonflikt M. Kiese gibt an, dass kein Interessenkonflikt besteht.

\section{Literatur}

Florida RL, Adler P, Mellander C (2017) The city as innovation machine. Reg Stud 51(1):86-96

IHK Ruhr (Industrie- und Handelskammern im Ruhrgebiet), Initiativkreis Ruhr, Wirtschaftsförderung Metropoleruhr $\mathrm{GmbH}$, RVR (2015) Resolution Wissenschaftsregion Ruhr. http://i-r.de/ fileadmin/redakteure/Bilder/Neuigkeiten/2015-09-16_Wissens gipfel_Ruhr/Resolution_Wissensgipfel.pdf. Zugegriffen: 26. März 2019

Kujath HJ (Hrsg) (2005) Knoten im Netz: Zur neuen Rolle der Metropolregionen in der Dienstleistungswirtschaft und Wissensökonomie. Stadt- und Regionalwissenschaften, 4. LIT, Münster

OECD (1996) The knowledge-based economy. OECD, Paris

Romer PM (1993) Two strategies for economic development: Using ideas and producing ideas. In: The World Bank (Hrsg) Proceedings of the world bank annual conference on development economics 1992. The World Bank, Washington, DC, S 63-91

Stehr N (1994) Arbeit, Eigentum und Wissen: Zur Theorie von Wissensgesellschaften. Suhrkamp, Frankfurt a.M.

Stehr N, Adolf M (2018) Ist Wissen Macht? Wissen als gesellschaftliche Tatsache, 2. Aufl. Velbrück, Weilerswist

UAR (Universitätsallianz Ruhr) (2019) Kompetenzfeld Metropolenforschung. http://metropolenforschung.uaruhr.de. Zugegriffen: 29. März 2019 